\title{
(Co)isotropic Pairs in Poisson and Presymplectic Vector Spaces
}

\author{
Jonathan LORAND ${ }^{\dagger}$ and Alan WEINSTEIN ${ }^{\ddagger}$ \\ † Department of Mathematics, ETH Zurich, Zurich, Switzerland \\ E-mail: jonathan.lorand@math.uzh.ch \\ URL: http://www.math.uzh.ch/?assistenten\&key1=11723 \\ $\ddagger$ Department of Mathematics, University of California, Berkeley, CA 94720 USA \\ E-mail: alanw@math.berkeley.edu \\ URL: http://math. berkeley.edu/ alanw/
}

Received March 01, 2015, in final form September 03, 2015; Published online September 10, 2015 http://dx.doi.org/10.3842/SIGMA.2015.072

\begin{abstract}
We give two equivalent sets of invariants which classify pairs of coisotropic subspaces of finite-dimensional Poisson vector spaces. For this it is convenient to dualize; we work with pairs of isotropic subspaces of presymplectic vector spaces. We identify ten elementary types which are the building blocks of such pairs, and we write down a matrix, invertible over $\mathbb{Z}$, which takes one 10 -tuple of invariants to the other.
\end{abstract}

Key words: coisotropic subspace; direct sum decomposition; Poisson vector space; presymplectic vector space

2010 Mathematics Subject Classification: 15A21; 18B10; 53D99

\section{Introduction}

The classification of pairs of linear coisotropic subspaces arises in many contexts, including the following two.

The first is the problem of classifying, up to conjugation by linear symplectomorphisms, canonical relations (lagrangian correspondences) from a finite-dimensional symplectic vector space to itself. Without symplectic structure, this classification of linear relations was carried out by Towber [13] and is a special case of results of Gel'fand and Ponomarev [5]. For graphs of symplectomorphisms, the classification amounts to identifying the conjugacy classes in the group of symplectic matrices. This classification and the problem of finding associated normal forms has a long history extending from Williamson [17] to Gutt [6]. In the general symplectic case, a result of Benenti and Tulczyjew [2, Proposizioni $4.4 \& 4.5$ ] tells us that any canonical relation $X \leftarrow Y$ is given by coisotropic subspaces of $X$ and $Y$ and a symplectomorphism between the corresponding reduced spaces. When $X=Y$, one step in the classification up to conjugacy of canonical relations is to classify the coisotropic pairs consisting of the range and domain. The further steps of the classification remain as work in progress. Partial results may be found in the first author's Master's thesis [8].

The second context is that of extending the Wehrheim-Woodward theory of linear canonical relations (see $[7,15]$ ) to the case where the category of lagrangian correspondences between symplectic vector spaces is enlarged to the category of coisotropic correspondences. Each pair of coisotropic subspaces of $X$ gives a WW morphism represented by a diagram having the form $\mathbf{1} \leftarrow X \leftarrow \mathbf{1}$, and isomorphic pairs represent the same WW morphism. There are also

\footnotetext{
${ }^{\star}$ This paper is a contribution to the Special Issue on Poisson Geometry in Mathematics and Physics. The full collection is available at http://www.emis.de/journals/SIGMA/Poisson2014.html
} 
nonisomorphic pairs representing the same WW morphism. The issue is to determine exactly which pairs are "WW equivalent". This problem is now solved, as part of a complete description of the WW categories of (co)isotropic relations (see [16]).

So far, we have been discussing symplectic ambient spaces. But since coisotropic correspondences are fundamental in Poisson geometry ${ }^{1}$, it is natural to consider the endomorphism classification and WW problems for linear coisotropic correspondences between general Poisson vector spaces. This leads immediately to the classification problem for coisotropic pairs in Poisson vector spaces, which is the subject of this paper ${ }^{2}$.

It turns out to be simpler to work indirectly with Poisson vector spaces via their duals, which are presymplectic (i.e., equipped with possibly degenerate skew-symmetric bilinear forms). The coisotropic subspaces are then replaced by their annihilators, which are isotropic. This duality gives an equivalence between the Poisson/coisotropic and presymplectic/isotropic categories. In the following, therefore, we will concentrate on pairs of isotropic subspaces in presymplectic vector spaces. To classify such pairs, we first show in Section 3 that any isotropic pair can be decomposed as the direct sum of pairs, each of which is of one of ten "elementary" types. In Section 4 we show that these elementary types are in turn isomorphic to multiples of ten indecomposable pairs in spaces of dimension 1,2 , and 3 , and that the multiplicities are invariants which fully classify isotropic pairs, up to conjugation by linear presymplectomorphisms. To prove that the multiplicities are invariant, we assemble them into a 10-vector and show that this vector is related by an invertible integer matrix to a 10-vector, each of whose entries is the dimension of a space constructed in a simple way from a presymplectic space and an ordered pair of its isotropic subspaces.

The preliminary version [9] of this paper contains our results in the symplectic case. We have in the meantime found the reference [12], which treats subspace pairs in spaces with bilinear forms, using methods and results related to quiver representations. The present paper gives an elementary and essentially self-contained treatment for spaces carrying a skew-symmetric form which is possibly degenerate; it remains for the future to relate our results to a broader representation-theoretic framework (see Remark 4.5).

\section{Preliminaries}

Throughout this paper, $V$ will denote a finite-dimensional presymplectic vector space, with presymplectic structure $\omega$. For any subspace $W \subseteq V$, we call the subspace $\{v \in V \mid \omega(v, w)=0$ $\forall w \in W\}$ the orthogonal of $W$ and denote it by $W^{\perp}$. For the radical $V^{\perp}$ of $V$ we reserve the letter $R$. A presymplectomorphism from $V$ to $\hat{V}$ is is a linear isomorphism $\varphi: V \rightarrow \hat{V}$ which pulls back the presymplectic structure on $\hat{V}$ to the one on $V$.

A subspace $A$ of $V$ is isotropic if $A \subseteq A^{\perp}$. An isotropic pair in $V$ is an ordered pair of isotropic subspaces in $V$. Isotropic pairs $(A, B)$ and $(\hat{A}, \hat{B})$ in $V$ and $\hat{V}$ respectively are equivalent if there exists a presymplectomorphism $\varphi: V \rightarrow \hat{V}$ such that $\varphi(A)=\hat{A}$ and $\varphi(B)=\hat{B}$. In the Poisson setting, where a coisotropic subspace is a subspace annihilated by an isotropic in the dual, this equivalence corresponds to there being an invertible Poisson map which takes one coisotropic pair to the other. In the symplectic situation, when $\omega$ is non-degenerate, any coisotropic subspace is the orthogonal of an isotropic subspace. Clearly, a linear symplectomorphism will take one coisotropic pair to the other if and only if it maps the corresponding isotropic orthogonals to one another.

An isotropic pair $(A, B)$ in $V$ is the direct sum of isotropic pairs $\left(A_{1}, B_{1}\right)$ and $\left(A_{2}, B_{2}\right)$ in $V_{1}$ and $V_{2}$, respectively, if $V=V_{1} \oplus V_{2}, A=A_{1} \oplus A_{2}, B=B_{1} \oplus B_{2}$, and $V_{1}$ is orthogonal to $V_{2}$

\footnotetext{
${ }^{1}$ See, e.g., [14], where these correspondences are called Poisson relations.

${ }^{2}$ The isomorphism classification of Poisson relations is treated in [10].
} 
with respect to $\omega .^{3}$ In this case, we have $\left(A_{i}, B_{i}\right)=\left(A \cap V_{i}, B \cap V_{i}\right)$, for $i=1,2$. The definition of direct sum of isotropic pairs naturally extends to any finite number of summands.

A decomposition

$$
V=\bigoplus_{i=1}^{m} V_{i}
$$

is orthogonal if its summands are pairwise orthogonal; it is distributive ${ }^{4}$ with respect to a subspace $W \subseteq V$ if

$$
W=\bigoplus_{i=1}^{m} W \cap V_{i} .
$$

The main task in Section 3 will be to construct an orthogonal decomposition of $V$ which is distributive with respect to isotropic subspaces $A$ and $B$, and such that each of the resulting summands has a simple form.

Remark 2.1. A general strategy for constructing a direct sum decomposition of $V$ which is distributive with respect to $W \subseteq V$ is the following stepwise procedure. In each step, find a subspace $V^{\prime} \subseteq V$ such that one of the following holds:

(i) $W \cap V^{\prime}=0$,

(ii) $W \cap V^{\prime}=V^{\prime}$.

If (i) is the case, then there exists a subspace $C \subseteq V$ such that $V=V^{\prime} \oplus C$ and $W \subseteq C$. This decomposition of $V$ is, by construction, distributive with respect to $W$. If (ii) is the case, i.e., $V^{\prime} \subseteq W$, then for any complement $C$ of $V^{\prime}$ in $V$ one has $W=V^{\prime} \oplus(W \cap C)$ by the modular law $^{\overline{5}}$, and so the resulting decomposition $V=V^{\prime} \oplus C$ is distributive with respect to $W$.

The following lemma shows that this procedure will indeed achieve the desired result. If in each step $V^{\prime}$ and $C$ can be chosen to be orthogonal, then the resulting decomposition will also be orthogonal.

Lemma 2.2. Suppose that $V$ has a decomposition (2.1), and let $W \subseteq V$ be a subspace. For each $l \in\{0,1, \ldots, m-1\}$ set

$$
C_{l}:=\bigoplus_{i=l+1}^{m} V_{i} .
$$

(i) If, for each $l \in\{1, \ldots, m-1\}$, the decomposition $V_{l} \oplus C_{l}$ is distributive with respect to $W \cap C_{l-1}$, then the decomposition (2.1) is distributive with respect to $W$.

(ii) If, for each $l \in\{1, \ldots, m-1\}$, the decomposition $V_{l} \oplus C_{l}$ is orthogonal, then the decomposition (2.1) is orthogonal.

\footnotetext{
${ }^{3}$ In other words, $\omega$ is the direct sum of the presymplectic structures on $V_{1}$ and $V_{2}$.

${ }^{4}$ It was pointed out to us by one of the referees that the existing language of graded vector spaces could be used here, but we find the "distributivity" terminology more convenient, because we make no use of any algebraic structure on the index set, and because it is superfluous to index the components in some decompositions. Of course, the basic properties associated to our definition correspond to basic facts about graded vector spaces.

${ }^{5}$ The modular law is the fact that, for subspaces $E, F$ and $G$ of a vector space $V$, if $E \subseteq G$, then $G \cap(E+F)=$ $E+(G \cap F)$. See [11, p. 56], for example.
} 
Proof. (i) We apply the assumptions successively to construct a decomposition of $W$ composed of the intersections of $W$ with the $V_{i}$. For $l=1$, by assumption we have a decomposition

$$
W=W \cap V_{1} \oplus W \cap C_{1} .
$$

The assumption for $l=2$, applied to the second summand of this decomposition, gives

$$
W=W \cap V_{1} \oplus W \cap V_{2} \oplus W \cap C_{2} .
$$

Clearly, proceeding in this manner for increasing $l$ will, after $m-1$ steps, lead to a decomposition

$$
W=W \cap V_{1} \oplus W \cap V_{2} \oplus \cdots \oplus W \cap V_{m-1} \oplus W \cap C_{m-1},
$$

which, after substitution using the identity $C_{m-1}=V_{m}$, is the desired result.

(ii) Choose any two indices $i, j \in\{1, \ldots, m\}$ such that $i \neq j$. We need to show that $V_{i}$ and $V_{j}$ are orthogonal. Because this relation is symmetric with respect to $i$ and $j$, we may assume without loss of generality that $i<j$. Then $i \leq m-1$ and $V_{j} \subseteq C_{i}$. By assumption $V_{i}$ is orthogonal to $C_{i}$, so in particular $V_{i}$ is orthogonal to $V_{j}$.

In the next lemma, we collect some basic properties of distributive decompositions. The proofs are, as above, straightforward; we leave them to the reader.

Lemma 2.3. Let $V=\bigoplus_{i=1}^{m} V_{i}$ be a decomposition which is distributive with respect to subspaces $E \subseteq V$ and $F \subseteq V$. Then,

(i) The decomposition is distributive with respect to $E \cap F$ and $E+F$, and

$$
(E+F) \cap V_{i}=\left(E \cap V_{i}\right)+\left(F \cap V_{i}\right)
$$

for each $i$.

(ii) If the decomposition of $V$ is orthogonal, then it is also distributive with respect to $E^{\perp}$, and

$$
E^{\perp} \cap V_{i}=\left(E \cap V_{i}\right)^{\perp} \cap V_{i}
$$

for each $i$.

Remark 2.4. As a special case of Lemma 2.3 (ii), with $V$ in the role of $E$, it follows that any orthogonal decomposition of $V$ is distributive with respect to the radical $R$.

We also recall some basic facts from presymplectic linear algebra.

Lemma 2.5. Let $E$ and $W$ be subspaces of $V$.

(i) If $E$ is symplectic, i.e., $\left.\omega\right|_{E}$ is non-degenerate, then $V=E \oplus E^{\perp}$.

(ii) If $E$ is such that $W=W \cap W^{\perp} \oplus E$, then $E$ is symplectic. In particular, any complement of $R$ in $V$ is symplectic.

(iii) $\operatorname{dim} W+\operatorname{dim} W^{\perp}=\operatorname{dim} V+\operatorname{dim} W \cap R$.

(iv) $W^{\perp \perp}=W+R$.

(v) When $V$ is symplectic, $W$ is lagrangian in $V$, i.e., $W^{\perp}=W$, if and only if $W$ is isotropic and $\operatorname{dim} W=\frac{1}{2} \operatorname{dim} V$.

We omit the proofs of these facts but use them to prove the following lemma which we will use later. 
Lemma 2.6. Let $I \subseteq V$ be an isotropic subspace, and let $L$ be a complement of $I \cap R$ in $I$. If $E$ is a complement of $R+I$ in $I^{\perp}$ and $L^{\prime}$ is a complement of $R+I$ in $E^{\perp}$, then $E$ and $L \oplus L^{\prime}$ are symplectic subspaces, $L$ is a lagrangian subspace of the latter, and $V=R \oplus E \oplus\left(L \oplus L^{\prime}\right)$.

Proof. All references in this proof are to Lemma 2.5 above. From (iv) one has that $I^{\perp \perp}=I+R$, and since $I$ is isotropic, $I+R \subseteq I^{\perp}$ (note that $R \subseteq I^{\perp}$ in any case). Thus, by (ii), with $I^{\perp}$ in the role of $W, E$ is symplectic. From this and (i) it follows that $V=E \oplus E^{\perp}$, and since by assumption $E^{\perp}=R \oplus L \oplus L^{\prime}$, one obtains $V=R \oplus E \oplus L \oplus L^{\prime}$. That $\left(L \oplus L^{\prime}\right)$ is symplectic follows from (ii), with $E^{\perp}$ in the role of $W$, since $E^{\perp} \cap\left(E^{\perp}\right)^{\perp}=E^{\perp} \cap(I+R)=R$ and $E^{\perp}=R \oplus\left(L \oplus L^{\prime}\right)$. Finally, to show that $L$ is lagrangian in $\left(L \oplus L^{\prime}\right)$, one can apply (iii), with $L$ in the role of $W$, which (using $L^{\perp}=I^{\perp}=R \oplus L \oplus E$ and $L \cap R=0$ ) shows that $2 \operatorname{dim} L=\operatorname{dim}\left(L \oplus L^{\prime}\right)$. By (v), this completes the proof.

\section{Decomposition of isotropic pairs}

Proposition 3.1. Any isotropic pair $(A, B)$ in a presymplectic space $V$ can be decomposed as an orthogonal direct sum of ten isotropic pairs, each of which is of an elementary type. If $\left(A_{i}, B_{i}\right)$ denotes the $i$-th summand, with ambient space $V_{i}$, the elementary types are:

1) $R_{1}=V_{1}, A_{1}=B_{1}=0$,

2) $R_{2}=V_{2}, A_{2}=B_{2}=V_{2}$,

3) $R_{3}=V_{3}, A_{3}=V_{3}$ and $B_{3}=0$,

4) $R_{4}=V_{4}, A_{4}=0$ and $B_{4}=V_{4}$,

5) $\operatorname{dim} V_{5}=3 \operatorname{dim} R_{5} ; R_{5}, A_{5}$ and $B_{5}$ have pairwise zero intersection, and $A_{5} \oplus R_{5}=B_{5} \oplus$ $R_{5}=A_{5} \oplus B_{5}$

6) $R_{6}=0, A_{6}$ and $B_{6}$ are lagrangian in $V_{6}$, and $A_{6}=B_{6}$,

7) $R_{7}=0, A_{7}$ is lagrangian in $V_{7}$ and $B_{7}=0$,

8) $R_{8}=0, A_{8}=0$ and $B_{8}$ is lagrangian in $V_{8}$,

9) $R_{9}=0, A_{9}$ and $B_{9}$ are lagrangian in $V_{9}$, and $A_{9} \cap B_{9}=0$,

10) $R_{10}=0, A_{10}=B_{10}=0$.

We will refer to these types as type 1 , type 2 , etc.

Proof. We will construct a ten-part orthogonal direct sum decomposition of $V$ by proceeding according to Remark 2.1, successively peeling away subspaces in such a way that, in each step, the resulting decomposition is both orthogonal and distributive with respect to $A$ and $B$, i.e., so that Lemma 2.2 will apply. At each step, we will find a summand $V_{i}$ such that $A_{i}=A \cap V_{i}$ and $B_{i}=B \cap V_{i}$ form a pair of type $i$ in $V_{i}$.

To begin, let $V_{1}$ be a complement of $R \cap(A+B)$ in $R$. Since $V_{1} \cap(A+B)=0$, we can choose a complement $C_{1}$ of $V_{1}$ in $V$ which contains $A+B$. Since $V_{1} \subseteq R$, the decomposition $V=V_{1} \oplus C_{1}$ is orthogonal.

Next, set $V_{2}=A \cap B \cap R \cap C_{1}$ and let $C_{2}$ be any complement of $V_{2}$ in $C_{1}$ (in particular then $A=V_{2} \oplus\left(A \cap C_{2}\right)$ and $\left.B=V_{2} \oplus\left(B \cap C_{2}\right)\right)$.

Now, let $V_{3}=A \cap R \cap C_{2}$; since $B \cap C_{2} \cap V_{3}=0$, we can choose a complement $C_{3}$ of $V_{3}$ in $C_{2}$ such that $B \cap C_{2} \subseteq C_{3}$. Set $V_{4}=B \cap R \cap C_{3}$. We have $A=V_{2} \oplus V_{3} \oplus A \cap C_{3}$ and $A \cap V_{4}=0$; in particular we can choose a complement $C_{4}$ of $V_{4}$ in $C_{3}$ which contains $A \cap C_{3} \subseteq C_{4}$. Since $V_{4} \subseteq B \cap C_{3}, B=V_{2} \oplus V_{4} \oplus B \cap C_{4}$. The decomposition $V=V_{1} \oplus V_{2} \oplus V_{3} \oplus V_{4} \oplus C_{4}$ is orthogonal, since the first four summands are subspaces of $R$. 
$C_{4}$ is now small enough so that $R \cap C_{4}$ has zero intersection with each of the isotropics $A \cap C_{4}$ and $B \cap C_{4}$; we consider $Q_{R}=R \cap C_{4} \cap(A+B)$, which is the radical of the presymplectic space $C_{4}$. One might try to find a complement to $Q_{R}$ which contains both $A \cap C_{4}$ and $B \cap C_{4}$, but this is not in general possible. Instead, we consider the space $S=A \cap C_{4} \cap\left(Q_{R}+B\right)+B \cap C_{4} \cap\left(Q_{R}+A\right)$, which contains $Q_{R}$ and $A \cap B \cap C_{4}$ as independent subspaces. Let $Q$ be a complement of $A \cap B \cap C_{4}$ in $S$ such that $Q_{R} \subset Q$, and set $Q_{A}=A \cap C_{4} \cap Q, Q_{B}=B \cap C_{4} \cap Q$. Note that each of the summands in the definition of $S$ decomposes as the direct sum of its intersections with $A \cap B \cap C_{4}$ and $Q$, and the latter subspaces span $Q$ and lie in $Q_{A}+Q_{B}$. This implies that $Q_{A}+Q_{B}=Q$. In fact, $Q=Q_{A} \oplus Q_{B}=Q_{R} \oplus Q_{A}=Q_{R} \oplus Q_{B}$. To see this, it suffices to show that $Q_{B} \subseteq Q_{A}+Q_{R}$, since we know that $Q_{R}, Q_{A}$ and $Q_{B}$ are pairwise independent, so dimension considerations then give the result. If $v=r+a$ is an element of $Q_{B} \subseteq B \cap C_{4} \cap\left(Q_{R}+A\right)$, with $r \in Q_{R}$ and $a \in A \cap C_{4}$, then $a=v-r$ must also lie in $Q$, since $v \in Q_{B} \subseteq Q$ and $r \in Q_{R} \subseteq Q$, which means that $a \in Q_{A}$, and thus $v \in Q_{R} \oplus Q_{A}$.

We will now use $Q$ to carry out the next step in our orthogonal decomposition of $V$. First, note that $Q^{\perp} \cap C_{4}=Q_{A}^{\perp} \cap C_{4}=Q_{B}^{\perp} \cap C_{4}$; in particular, it follows that $Q^{\perp} \cap C_{4}$ contains $A \cap C_{4}+B \cap C_{4}$. In this latter space, let $T$ be a complement of $A \cap B \cap C_{4}$ which contains $Q=Q_{A} \oplus Q_{B}$. Then, in particular, $A \cap C_{4}=\left(A \cap B \cap C_{4}\right) \oplus\left(A \cap C_{4} \cap T\right)$, with $Q_{A} \subseteq A \cap C_{4} \cap T$, and $B \cap C_{4}=\left(A \cap B \cap C_{4}\right) \oplus\left(B \cap C_{4} \cap T\right)$, with $Q_{B} \subseteq B \cap C_{4} \cap T$. Let $A^{\prime}$ and $B^{\prime}$ be complements of $Q_{A}$ and $Q_{B}$ in $A \cap C_{4} \cap T$ and $B \cap C_{4} \cap T$, respectively. Then, $A^{\prime}$ and $B^{\prime}$ are independent, and their direct sum is a complement of $Q$ in $T$. This gives a decomposition

$$
A \cap C_{4}+B \cap C_{4}=Q_{A} \oplus Q_{B} \oplus\left(A \cap B \cap C_{4}\right) \oplus A^{\prime} \oplus B^{\prime} .
$$

Now choose a complement $C_{5}$ of $Q$ in $Q^{\perp} \cap C_{4}$ which contains the last three summands in (3.1), and let $P$ be a complement of $Q$ in $C_{5}^{\perp} \cap C_{4}$. By Lemma 2.6, with $Q_{A}$ in the role of $I$ (recall that $\left.Q_{A}^{\perp} \cap C_{4}=Q^{\perp} \cap C_{4}\right)$, we have an orthogonal decomposition $C_{4}=Q_{R} \oplus\left(Q_{A} \oplus P\right) \oplus C_{5}$, the spaces $C_{5}$ and $\left(Q_{A} \oplus P\right)$ are symplectic, and $Q_{A}$ is lagrangian in the latter. We set $V_{5}=Q_{R} \oplus Q_{A} \oplus P$. By construction, the decomposition $C_{4}=V_{5} \oplus C_{5}$ is distributive with respect to both $A \cap C_{4}$ and $B \cap C_{4}$ : indeed $Q_{A} \subseteq A \cap V_{5}$ and $\left(A \cap B \cap C_{4}\right) \oplus A^{\prime} \subseteq A \cap C_{5}$ form a decomposition of $A \cap C_{4}$, so the inclusions are in fact equalities; this is similarly the case for $Q_{B} \subseteq B \cap V_{5}$ and $\left(A \cap B \cap C_{4}\right) \oplus B^{\prime} \subseteq B \cap C_{5}$ in $B \cap C_{4}$.

At this point we have completely decomposed the radical $R$; it remains to decompose the symplectic space $C_{5}$ with respect to the isotropics $A \cap C_{5}$ and $B \cap C_{5}$. Let $C_{6}$ be a complement of $A \cap B \cap C_{5}$ in $(A \cap B)^{\perp} \cap C_{5}$; since $A \cap C_{5}$ and $B \cap C_{5}$ each contain $A \cap B \cap C_{5}$ and lie in $(A \cap B)^{\perp} \cap C_{5}$, they each can be decomposed as the sum of $A \cap B \cap C_{5}$ and their respective intersections with $C_{6}$. Choose a complement of $A \cap B \cap C_{5}$ in $C_{6}^{\perp} \cap C_{5}$; by Lemma 2.6 it is nondegenerately paired with $A \cap B \cap C_{5}$, and the direct sum of these two spaces forms a symplectic space $V_{6}$ in which $A \cap B \cap C_{5}$ is lagrangian.

Next, consider $A \cap B^{\perp} \cap C_{6}$ and let $C_{7}$ be a complement of this subspace in $\left(A \cap B^{\perp}\right)^{\perp} \cap C_{6}$. Since $A \cap B^{\perp} \cap B \cap C_{6}=0$ and $B \cap C_{6} \subseteq\left(A \cap B^{\perp}\right)^{\perp} \cap C_{6}$, we can choose $C_{7}$ so as to contain $B \cap C_{6}$. By Lemma 2.6 again, any complement to $A \cap B^{\perp} \cap C_{6}$ in $C_{7}^{\perp} \cap C_{6}$ is non-degenerately paired with $A \cap B^{\perp} \cap C_{6}$, forming a symplectic subspace $V_{7}$ in which $A \cap B^{\perp} \cap C_{6}$ is a lagrangian subspace.

This same construction can now be applied in $C_{7}$, with the roles of $A$ and $B$ exchanged, to yield an orthogonal decomposition $C_{7}=V_{8} \oplus C_{8}$ into symplectic subspaces which is distributive with respect to $A \cap C_{7}$ and $B \cap C_{7}$ and is such that $B \cap A^{\perp} \cap C_{7}$ is a lagrangian subspace of $V_{8}$.

In the symplectic space $C_{8}, A \cap C_{8}$ and $B \cap C_{8}$ are independent isotropic subspaces such that $A \cap B^{\perp} \cap C_{8}=B \cap A^{\perp} \cap C_{8}=0$. We claim that $V_{9}=\left(A \cap C_{8}\right) \oplus\left(B \cap C_{8}\right)$ and $V_{10}=A^{\perp} \cap B^{\perp} \cap C_{8}$ form an orthogonal decomposition $V_{8}=V_{9} \oplus V_{10}$ into symplectic subspaces. For this, it is sufficient to show that $V_{9}$ and $V_{10}$ are independent, since $V_{10}=V_{9}^{\perp} \cap C_{8}$. So suppose $v \in V_{9} \cap V_{10}$. As an element of $V_{9}, v=a+b$, with $a \in A \cap C_{8}$ and $b \in B \cap C_{8}$. Since $A \cap C_{8} \subseteq A^{\perp} \cap C_{8}, a$ is 
an element of $A^{\perp} \cap C_{8}$, as is $v$. Thus $b=v-a$ lies in $B \cap A^{\perp} \cap C_{8}=0$. The same argument shows that $a=0$, and hence $v=0$, as desired. Finally, we check that $A \cap C_{8}$ and $B \cap C_{8}$ are each lagrangian subspaces of $V_{9}$ : on the one hand, being isotropic, they each may have at most half the dimension of $V_{9}$; on the other hand, their direct sum spans $V_{9}$, so they must each have dimension $\frac{1}{2} \operatorname{dim} V_{9}$, i.e., they are each lagrangian.

This completes our proof, since by Lemma 2.2 the constructed decomposition of $V$ is both orthogonal and distributive with respect to $A$ and $B$ (and also $R$, cf. Remark 2.4), and it is clear from the construction that the intersections of these spaces with the summands $V_{i}$ each define isotropic pairs of the types stated in the proposition.

\section{Indecomposables}

We denote the canonical basis vectors in $\mathbb{R}^{n}$ by $e_{1}, e_{2}, \ldots, e_{n}$. Angled brackets ' \langle\rangle ' indicate the span of a vector or a list of vectors. The standard (pre)symplectic structure on $\mathbb{R}^{n}$ of rank $2 k$ is the bilinear form given in canonical coordinates by the $n \times n$ matrix

$$
\left(\begin{array}{ccc}
0 & \mathbb{I}_{k} & 0 \\
-\mathbb{I}_{k} & 0 & 0 \\
0 & 0 & 0
\end{array}\right),
$$

where $\mathbb{I}_{k}$ denotes the $k \times k$ identity matrix, and the zeros denote square zero matrices of appropriate sizes. A symplectic basis of a vector space with symplectic form $\omega$ is one such that the coordinate matrix of $\omega$ with respect to this basis has the same form as the matrix of the standard symplectic form.

Definition 4.1. An isotropic pair $(A, B)$ in $V$ is indecomposable if for any direct sum decomposition into isotropic pairs $\left(A_{1}, B_{1}\right)$ and $\left(A_{2}, B_{2}\right)$, corresponding to an orthogonal decomposition $V=V_{1} \oplus V_{2}$, it follows that either $V_{1}=0$ or $V_{2}=0$.

Theorem 4.2. Any indecomposable isotropic pair $(A, B)$ in a presymplectic space $V$ is isomorphic to one of the following isotropic pairs.

Pairs where the ambient space is $\mathbb{R}$, carrying the zero presymplectic structure:

1) $A=0, B=0$,

2) $A=\left\langle e_{1}\right\rangle, B=\left\langle e_{1}\right\rangle$,

3) $A=\left\langle e_{1}\right\rangle, B=0$,

4) $A=0, B=\left\langle e_{1}\right\rangle$.

Pairs where the ambient space is $\mathbb{R}^{3}$, equipped with the standard presymplectic structure of rank 2:

5) $A=\left\langle e_{1}\right\rangle, B=\left\langle e_{1}+e_{3}\right\rangle$.

Pairs where the ambient space is $\mathbb{R}^{2}$, carrying the standard symplectic structure:

6) $A=\left\langle e_{1}\right\rangle, B=\left\langle e_{1}\right\rangle$,

7) $A=\left\langle e_{1}\right\rangle, B=0$,

8) $A=0, B=\left\langle e_{2}\right\rangle$,

9) $A=\left\langle e_{1}\right\rangle, B=\left\langle e_{2}\right\rangle$,

10) $A=0, B=0$. 
Any isotropic pair can be decomposed as the direct sum of indecomposable isotropic pairs. The multiplicities $n_{1}, \ldots, n_{10}$ of the indecomposable types in the decomposition are invariants of the isotropic pair itself. Two isotropic pairs are equivalent if and only if their corresponding multiplicities are equal.

Proof. First of all, we check that each of the isotropic pairs listed above is indeed indecomposable. For types 1 through 4 and 6 through 10, this follows directly from dimension considerations and, in the latter cases, orthogonality. In the case of type 5 , suppose that there exists a non-trivial orthogonal decomposition of this isotropic pair into two summands, with ambient spaces $V_{1}$ and $V_{2}$ respectively. Since $\operatorname{dim} V=3$, we may assume without loss of generality that $\operatorname{dim} V_{1}=1$. Then $V_{1}$ is isotropic, and so its radical $R_{1}$ is equal to $V_{1}$. Since $R_{1}=R \cap V_{1}$ (cf. Remark 2.4) and $\operatorname{dim} R=1$, we find that $R=V_{1}$. Because $A, B$, and $R$ are pairwise independent, it follows that $A \subseteq V_{2}$ and $B \subseteq V_{2}$ and, in turn, $A \oplus B \subseteq V_{2}$. But $R \subseteq A \oplus B$, which implies $R \subseteq V_{2}$, a contradiction to $R \subseteq V_{1}$.

The fact that these are all the indecomposable types follows from the decomposability to be proved next.

To prove that any isotropic pair can be decomposed as an orthogonal direct sum of indecomposables of the above types, it is enough to show that any elementary isotropic pair of type $i$, where $i \in\{1, \ldots, 10\}$, is isomorphic to the orthogonal direct sum of copies of the $i$ 'th indecomposable pair above. This is straightforward when the presymplectic structure is either non-degenerate or zero (in the former case one can choose a symplectic basis adapted to the isotropic subspaces, and in the latter any choice of basis will do the job). We leave the details of this argument to the reader and focus on the case when $(A, B)$ is of type 5 . In this case, we know that $\operatorname{dim} V=3 n$ for some $n \in \mathbb{N}$ and that $A \oplus B=R \oplus A=R \oplus B$. Let $Q$ denote the subspace $A \oplus B$ and let $U$ be a complement of $R$ in $V$ such that $A \subseteq U$. For dimension reasons, $A$ is lagrangian in the symplectic subspace $U$; let $P$ be a lagrangian complement of $A$ in $U$.

Because $B \subseteq R \oplus A$, if $\left\{b_{1}, \ldots, b_{n}\right\}$ is a basis of $B$, each $b_{i}$ has a unique decomposition

$$
b_{i}=r_{i}+a_{i}
$$

with $r_{i} \in R \backslash\{0\}$ and $a_{i} \in A \backslash\{0\}$. It is a routine exercise to show that $\left\{r_{1}, \ldots, r_{n}\right\}$ and $\left\{a_{1}, \ldots, a_{n}\right\}$ are bases of $R$ and $A$, respectively. Since $P$ is a lagrangian complement of $A$ in $U$, one can find a basis $\left\{p_{1}, \ldots, p_{n}\right\}$ of $P$ which together with $\left\{a_{1}, \ldots, a_{n}\right\}$ forms a symplectic basis of $U$. For each $i$, set $R_{i}:=\left\langle r_{i}\right\rangle, A_{i}:=\left\langle a_{i}\right\rangle, B_{i}:=\left\langle b_{i}\right\rangle, P_{i}:=\left\langle p_{i}\right\rangle, Q_{i}:=\left\langle r_{i}, a_{i}\right\rangle$, and $U_{i}:=\left\langle a_{i}, p_{i}\right\rangle$. Because $R, A, B$ and $P$ are pairwise independent, the $R_{i}, A_{i}, B_{i}$ and $P_{i}$ are too, and from (4.1) it follows that $Q_{i}=R_{i} \oplus A_{i}=R_{i} \oplus B_{i}=A_{i} \oplus B_{i}$. Thus, for each $i,\left(A_{i}, B_{i}\right)$ is an indecomposable isotropic pair of type 5 in the presymplectic space $V_{i}=R_{i} \oplus U_{i}$. From the properties of a symplectic basis, it follows that $U=U_{1} \oplus \cdots \oplus U_{n}$ is an orthogonal decomposition, and because each $R_{i}$ is orthogonal to all of $V$, the decomposition $V=V_{1} \oplus \cdots \oplus V_{n}$ is orthogonal as well.

To show that the multiplicities of the indecomposable types in a decomposition are invariants of the pair being decomposed, we first write down a set of invariants associated to any (in general decomposable) isotropic pair $(A, B)$ in presymplectic $V$ :

$$
\begin{array}{llrl}
k_{1}=\frac{1}{2}(\operatorname{dim} V-\operatorname{dim} R), & & k_{6}=\operatorname{dim} R \cap A, \\
k_{2}=\operatorname{dim} R, & & k_{7}=\operatorname{dim} R \cap B, \\
k_{3}=\operatorname{dim} A, & & k_{8}=\operatorname{dim} R \cap A \cap B, \\
k_{4}=\operatorname{dim} B, & & k_{9}=\operatorname{dim} R \cap(A+B), \\
k_{5}=\operatorname{dim} A \cap B, & & k_{10}=\operatorname{dim} A^{\perp} \cap B .
\end{array}
$$

Given a decomposition of $(A, B)$ into indecomposables, one can group together all indecomposables of each type to obtain a ten-part decomposition of $(A, B)$ into elementary types, as 
in Proposition 3.1. By Lemma 2.3, this decomposition is distributive with respect to each of the subspaces in (4.2), so each has a corresponding decomposition. Accordingly, the column vector $\mathbf{k}$ of invariants is obtained from the column vector $\mathbf{n}$ of multiplicities by multiplication by the matrix

$$
\mathbf{M}=\left(\begin{array}{llllllllll}
0 & 0 & 0 & 0 & 1 & 1 & 1 & 1 & 1 & 1 \\
1 & 1 & 1 & 1 & 1 & 0 & 0 & 0 & 0 & 0 \\
0 & 1 & 1 & 0 & 1 & 1 & 1 & 0 & 1 & 0 \\
0 & 1 & 0 & 1 & 1 & 1 & 0 & 1 & 1 & 0 \\
0 & 1 & 0 & 0 & 0 & 1 & 0 & 0 & 0 & 0 \\
0 & 1 & 1 & 0 & 0 & 0 & 0 & 0 & 0 & 0 \\
0 & 1 & 0 & 1 & 0 & 0 & 0 & 0 & 0 & 0 \\
0 & 1 & 0 & 0 & 0 & 0 & 0 & 0 & 0 & 0 \\
0 & 1 & 1 & 1 & 1 & 0 & 0 & 0 & 0 & 0 \\
0 & 1 & 0 & 1 & 1 & 1 & 0 & 1 & 0 & 0
\end{array}\right)
$$

which has unit determinant and is thus invertible over $\mathbb{Z}$. Hence, the multiplicities $\mathbf{n}$ can be recovered from the invariants $\mathbf{k}$ via the inverse of $\mathbf{M}$ and are themselves invariants; i.e., decompositions of equivalent pairs into indecomposables lead to the same multiplicities.

Remark 4.3. The set of possible vectors $\mathbf{n}$ of multiplicities is of course $\mathbb{Z}_{\geq 0}^{10}$; the set of possible vectors $\mathbf{k}$ is a subset of $\mathbb{Z}_{\geq 0}^{10}$ constrained only by the ten inequalities arising from the condition that $\mathbf{n} \in \mathbb{Z}_{\geq 0}^{10}$.

Remark 4.4. The first 9 invariants in (4.2) solve the "triple of subspaces problem" of classifying, up to linear isomorphism, three arbitrary linear subspaces $R, A, B$ of a vector space $V$ (without presymplectic structure). For such subspace triples, all indecomposable types are 1-dimensional, except for one type which is 2 -dimensional. This 2 -dimensional type is closely related to the 5 th indecomposable type of isotropic pair listed in Theorem $4.2 .{ }^{6}$

The tenth invariant $k_{10}$ in (4.2) carries the essential information (other than $R$ ) connected with the presymplectic structure. For this invariant one can just as well choose $\operatorname{dim} A^{\perp} \cap B$; this shows, in particular, that the isotropic pairs $(A, B)$ and $(B, A)$ are equivalent when $\operatorname{dim} A=$ $\operatorname{dim} B,{ }^{7}$ since all other invariants are symmetric with respect to $A$ and $B$.

Remark 4.5. The elementary types listed in Proposition 3.1 are like the isotypic components in group representations. The invariants (4.2) are therefore, in some sense, analogous to characters, which are linear functions of the multiplicities, and which determine a representation up to equivalence in many situations. We wonder whether the present results might be recovered, for example, from the theory of some general class of quiver representations, perhaps by relations rather than maps. Note that Theorem 4.2 is a Krull-Schmidt type statement, in spirit similar to Theorem 2.19 in [4], for example. It could probably also be framed in more abstract terms, such as in [1].

The ten types of indecomposable isotropic pairs given in Theorem 4.2 can be visualized as follows ( $A$ is green, $B$ is blue, $R$ is red):

\footnotetext{
${ }^{6}$ For the classification of subspace triples, see Brenner [3, pp. 109-110], and Etingof et al. [4, pp. 84-86].

${ }^{7}$ This is the case, for example, in the symplectic case when the corresponding coisotropics $A^{\perp}$ and $B^{\perp}$ are the range and domain respectively of a linear canonical relation in $V \times \bar{V}$.
} 


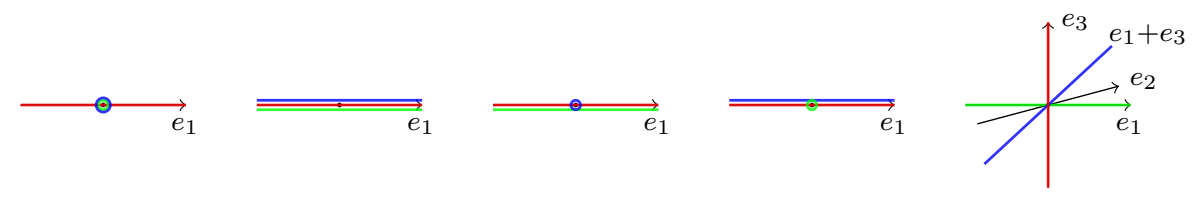

Type $1 \quad$ Type $2 \quad$ Type $3 \quad$ Type $4 \quad$ Type 5

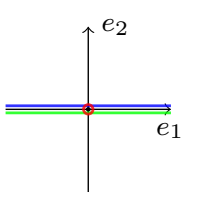

Type 6

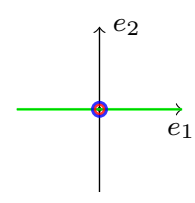

Type 7

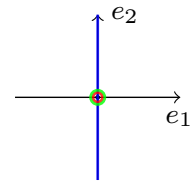

Type 8

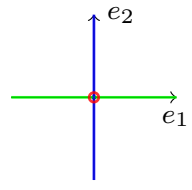

Type 9

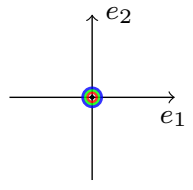

Type 10

\section{Acknowledgements}

Jonathan Lorand was partially supported by ETH Zurich, the city of Zurich, and the Anna \& Hans Kägi Foundation. Part of this research was conducted while he was at UC Berkeley as a Visiting Student Researcher. The authors wish to thank the referees, in particular for comments which led to a more concise presentation of our results.

\section{References}

[1] Atiyah M.F., On the Krull-Schmidt theorem with application to sheaves, Bull. Soc. Math. France 84 (1956), 307-317.

[2] Benenti S., Tulczyjew W., Symplectic linear relations, Mem. Accad. Sci. Torino Cl. Sci. Fis. Mat. Natur. (5) 5 (1981), 71-140.

[3] Brenner S., Endomorphism algebras of vector spaces with distinguished sets of subspaces, J. Algebra 6 (1967), 100-114.

[4] Etingof P., Golberg O., Hensel S., Liu T., Schwendner A., Vaintrob D., Yudovina E., Introduction to representation theory, Lecture notes, available at http://www-math.mit.edu/ etingof/replect.pdf.

[5] Gel'fand I.M., Ponomarev V.A., Problems of linear algebra and classification of quadruples of subspaces in a finite-dimensional vector space, in Hilbert Space Operators and Operator Algebras (Proc. Internat. Conf., Tihany, 1970), Colloq. Math. Soc. János Bolyai, Vol. 5, North-Holland, Amsterdam, 1972, $163-237$.

[6] Gutt J., Normal forms for symplectic matrices, Port. Math. 71 (2014), 109-139, arXiv:1307.2403.

[7] Li-Bland D., Weinstein A., Selective categories and linear canonical relations, SIGMA 10 (2014), 100, 31 pages, arXiv:1401.7302.

[8] Lorand J., Classifying linear canonical relations, arXiv:1508.04568.

[9] Lorand J., Weinstein A., Coisotropic pairs, arXiv:1408.5620.

[10] Lorand J., Weinstein A., Decomposition of (co)isotropic relations, in preparation.

[11] Roman S., Advanced linear algebra, Graduate Texts in Mathematics, Vol. 135, 3rd ed., Springer, New York, 2008.

[12] Sergeichuk V.V., Classification of pairs of subspaces in spaces with scalar product, Ukrainian Math. J. 42 (1990), 487-491.

[13] Towber J., Linear relations, J. Algebra 19 (1971), 1-20.

[14] Weinstein A., Coisotropic calculus and Poisson groupoids, J. Math. Soc. Japan 40 (1988), 705-727.

[15] Weinstein A., A note on the Wehrheim-Woodward category, J. Geom. Mech. 3 (2011), 507-515, arXiv:1012.0105.

[16] Weinstein A., Categories of (co)isotropic linear relations, arXiv:1503.06240.

[17] Williamson J., On the normal forms of linear canonical transformations in dynamics, Amer. J. Math. 59 (1937), 599-617. 